\title{
Surface strain distribution in isolated single lumbar vertebrae
}

\author{
J. S. SHAH, J. COGGINS, R. ROGERS, M. I. V. JAYSON, AND W. G. J. HAMPSON \\ From H. H. Wills Physics Laboratory and Department of Medicine, University of Bristol; \\ Royal National Hospital for Rheumatic Diseases, Bath; and Winford Orthopaedic Hospital
}

\begin{abstract}
Shah, J. S., Coggins, J., Rogers, R., Jayson, M. I. V., and Hampson, W. G. J. (1976). Annals of the Rheumatic Diseases, 35, 51-55. Surface strain distribution in isolated single lumbar vertebrae. The surface strains of isolated single lumbar vertebrae under compressive load were examined by the techniques of brittlecoat and photoelastic analysis. Directions of principal strain components have been determined. Areas of stress concentration were found around the vertebral rims, near the pedicles, and around the foramina of the basivertebral veins.
\end{abstract}

The human spine in life is subjected to a bewildering combination of shear, torsion, compression, and tensile forces. This paper attempts to identify the principal directions of strain and areas of high strain concentration in isolated vertebrae loaded in a simple compressive force system. While accepting that the experimental conditions are vastly different from those occurring in vivo, such knowledge may assist in the understanding of the mechanical role of the spine, and indicate ways in which stress-induced damage arises.

\section{Methods}

Two techniques, brittlecoat analysis and photoelastic analysis, were used for the assessment of strain.

\section{BRITTLECOAT ANALYSIS}

In this established engineering method a thin layer of brittlecoat is applied to the surface of the specimen under test. This coating is designed to fail at much lower stresses than the specimen itself and the resulting cracks appear at right-angles to the tension strain, thereby showing their direction on the surface of the specimen. Compression strains can also be studied by loading the specimen for 2 hours during which time the brittlecoat relaxes and conforms to the altered vertebral shape. Sudden removal of the load will then produce new cracks at right-angles to the original compression strain.

Cadaveric lumbar vertebrae were prepared by 3 hours' boiling in sodium perborate solution, which allows easy removal of all soft tissue. The perfectly cleaned and dried vertebrae were then sprayed with a metallized undercoat, followed by a thin layer of brittlecoat. Each prepared vertebra was loaded parallel to the axis of the vertebral body in a Dennison Tensometer adapted for compression, the force being applied through rubber pads cut in the shape of intervertebral discs. Cracks representing principal tension strain immediately appeared and were noted and photographed. After 2 hours the load was removed and new cracks representing compression strain were similarly recorded.

\section{PHOTOELASTIC ANALYSIS}

This technique relies on the principle that strains in a transparent object can be seen as colour fringes if viewed between crossed polarizing filters (Maxwell, 1853). By observing these colour fringes it is possible to locate highly strained areas and determine the directions of principal strains. Normally the strains and therefore the fringes will disappear on removal of an external load. However, by using a technique of 'stress freezing' (detailed below) this information can be 'locked-in' for subsequent examination.

Accurate models of lumbar vertebrae were cast using Araldite CT 200 (Ciba Ltd.) inside previously prepared Silcoset (ICI Ltd.) moulds. The vertebral bodies were loaded in the same manner as the brittlecoat specimens, except that when under load they were heated in a miniature furnace to $120^{\circ} \mathrm{C} \pm 1^{\circ} \mathrm{C}$. By subsequently cooling the models at a rate of $2^{\circ} \mathrm{C}$ per hour while still under load, the stresses were 'locked-in'. Slices cut from the vertebral model could later be examined to determine the strain directions near the surface of the model.

\section{Results}

\section{BRITTLECOAT ANALYSIS}

Six lumbar spines were removed at post mortem from previously healthy adults, all under the age of 45 years, who had died shortly after road accidents. Six 

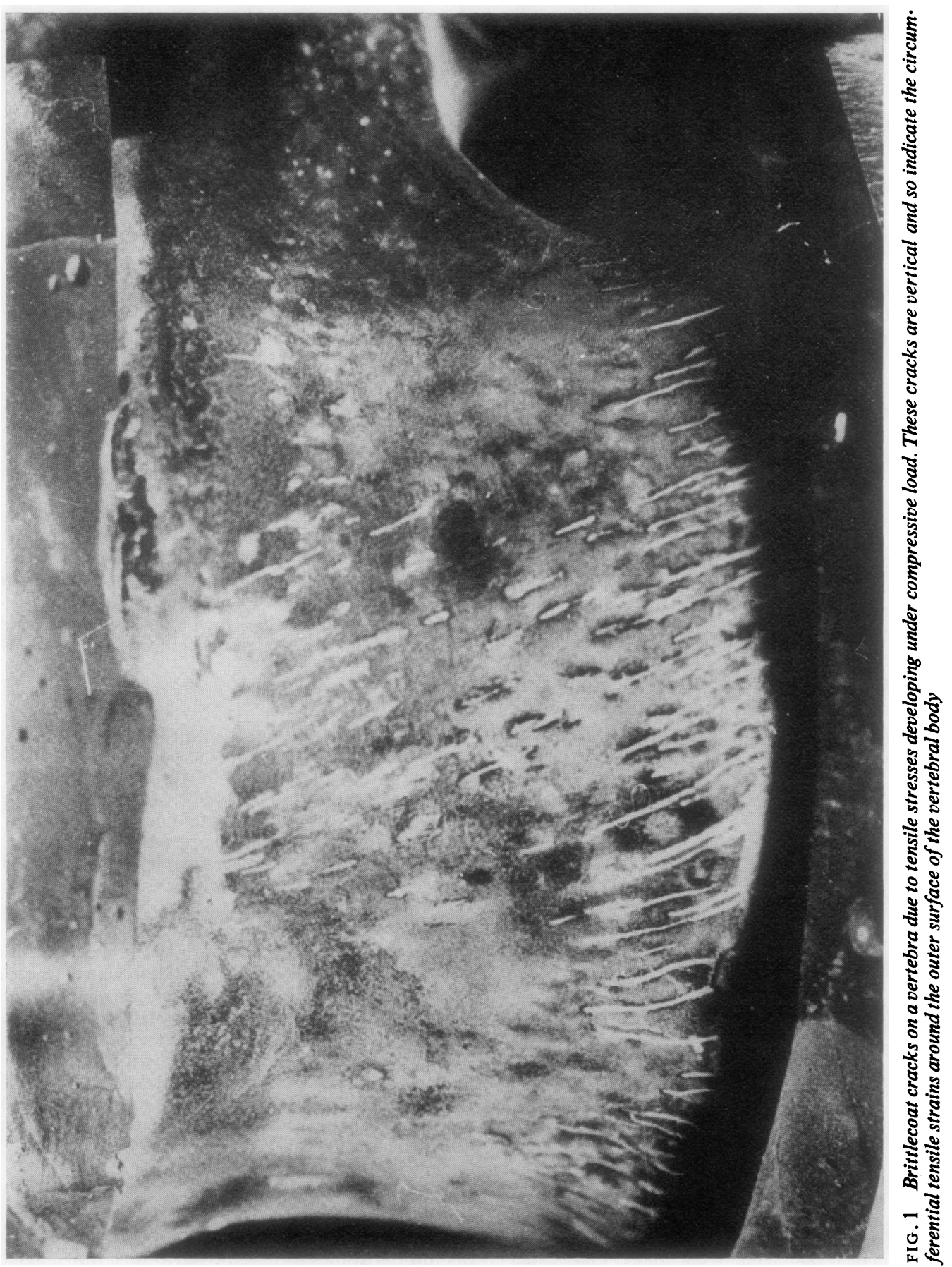
3rd lumbar, six 4th lumbar, and six 5th lumbar vertebrae were therefore available for examination.

Fig. 1 shows a typical example of cracks which appear on compressive loading. The cracks are vertically inclined and indicate circumferential tensile strains around the outer surface of the vertebral body. Fig. 2 shows a typical example of cracks which appear when compression has been applied for 2 hours and the applied force is subsequently removed. These cracks are circumferential and indicate vertical compression strains. Fig. $3 a$ and $b$ are composite diagrams of the tensile and compressive strain directions from all 18 vertebrae. They show high strain areas towards the upper and lower surfaces of the vertebral bodies, the roots of the pedicles, and around the foramina of the basivertebral veins.

\section{PHOTOELASTIC ANALYSIS}

Models were made from the 4th and 5th lumbar vertebrae of a 35 -year-old male patient whose spine had already been used for the brittlecoat experiments. After the strain directions had been 'locked-in', slices were cut from near the surface of the models (Fig. 4). The fringes produced in each slice were examined and from them the strain trajectories determined (Fig. 5). Intensive strain concentrations were again noted around the roots of the pedicles and around the foramina of the basivertebral veins. The strain levels near the outer surfaces of the vertebral bodies were higher towards the vertebral end plates.

\section{Discussion}

Complex stresses develop in the living lumbar spine and might be important in the development of disc generation. Farfan and others (1970) for example, have examined the contribution of torsion. In this study isolated lumbar vertebrae were loaded in a simple compression force system. Therefore any conclusions may only be taken as a tentative guide to the likely stress/strain distributions which occur in life. Since dead bone treated with special materials and Araldite models were used, the elastic properties of the specimens differed from those of living vertebrae so that the absolute levels of stresses cannot be calculated. However, since both brittlecoat and photoelastic analysis give the relative distribution of strains (and therefore relative stresses) we can identify

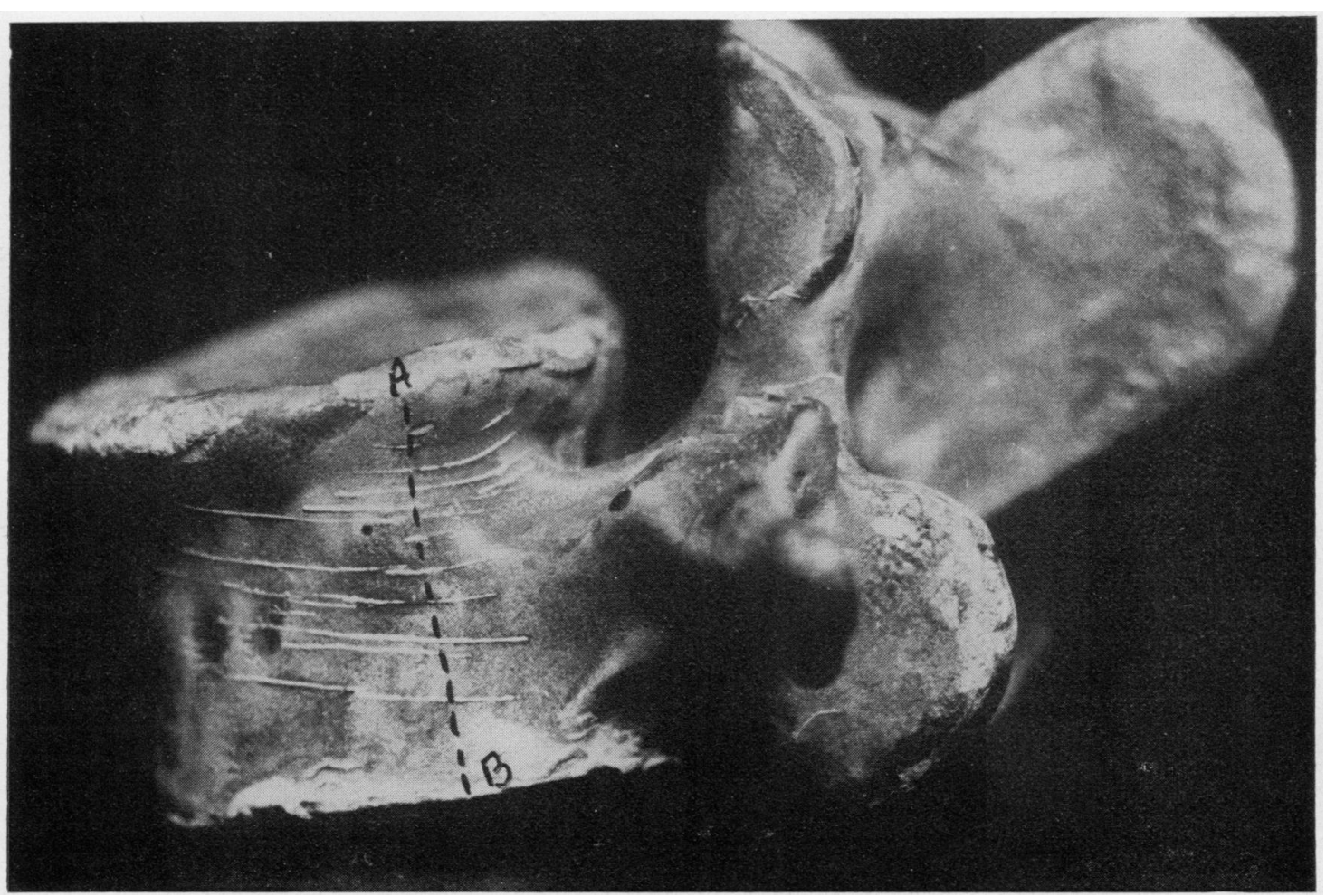

FIG. 2 Brittlecoat cracks on a vertebra observed after removal of the compressive force. A-B shows the direction of the applied force. These cracks are circumferential and indicate the vertical compressive strains developed under compressive load 

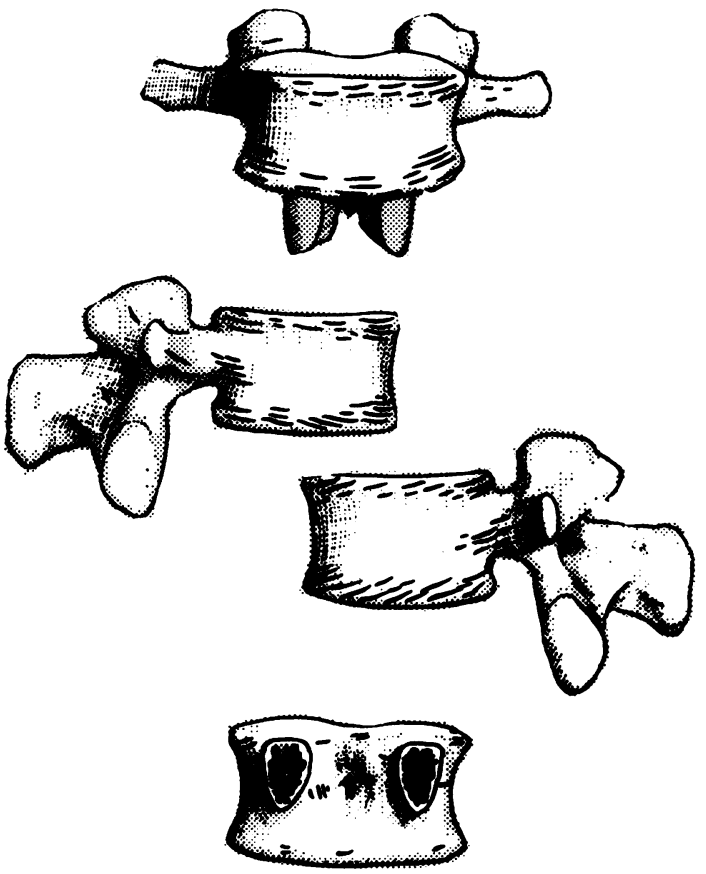

$a$
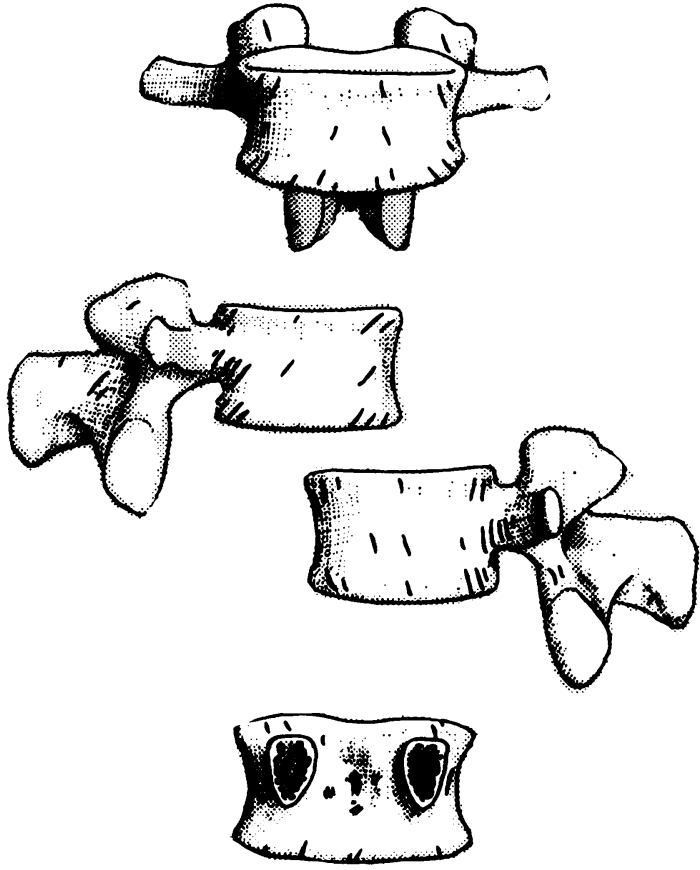

b

FIG. 3

(a) A composite diagram showing the common sites and directions of the principal tensile strains. These strains are horizontally inclined being at right angles to the cracks shown in Fig. 1 and are most marked towards the upper and lower surfaces of the vertebral bodies and in the pedicles.

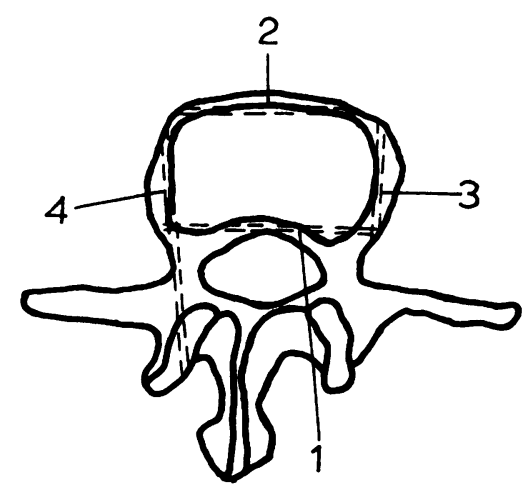

FIG. 4 Showing the sites where slices were taken for photoelastic analysis

the areas of stress concentration in the vertebrae under simple compressive loads.

Gallois and Japiot (1925) classified the trabecular architecture of vertebrae and found a dense system of (b) A composite diagram of the common sites and directions of the principal compressive strains. These strains are vertically inclined being at right angles to the cracks shown in Fig. 2. Overall they show some concentration towards the roots of the pedicles, the upper and lower vertebral surfaces, and around the foramina of the basivertebral veins

oblique trabeculae supporting the pedicles. This correlates with our findings of dense concentrations at these sites. This could be an example of the statement by Wolff (1870) that bone becomes adapted during growth to the mechanical forces acting on it.

Vernon-Roberts and Pirie (1973) recorded the distribution of trabecular microfractures within the bodies of lumbar vertebrae. They found the greatest number of trabecular lesions in the upper and lower thirds of the vertebral bodies. Although their study examined the internal trabecular structure, whereas the present examination was directed at stresses near the surface, both studies suggest that the major stresses are towards the upper and lower surfaces of the vertebral bodies. It is possible that the high stresses play some part in the genesis of osteophytes since these develop at the vertebral rims.

We thank Mr. F. Bannister and Mr. S. Rundle for technical assistance. This work was performed with the assistance of a grant from the Arthritis and Rheumatism Council. 

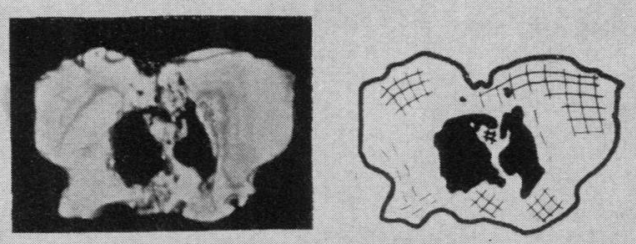

1

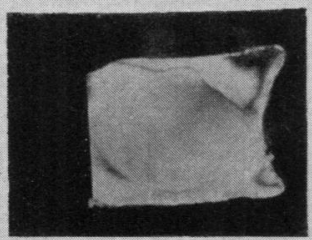

2

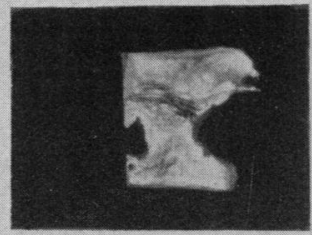

3

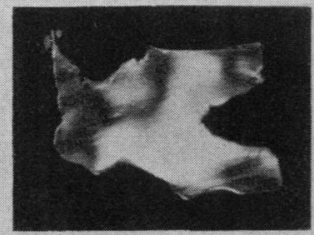

4

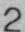

1

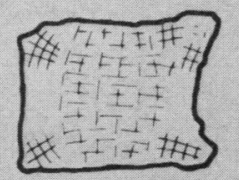

2

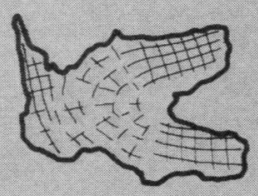

4

FIG. 5 The left-hand column shows typical photoelastic fringe patterns obtained from the vertebral slices. The righthand column shows the sites and directions of the calculated principal stress trajectories obtained by photoelastic analysis

\section{References}

Farfan, H. F., Cossette, J. W., Robertson, G. H., Wells, R. V., and Kraus, H. (1970) J. Bone Jt Surg., 52A, 468 (The effects of torsion on the lumbar intervertebral joints: the role of torsion in the production of disc degeneration)

GALlOIS AND JAPIOT (1925) Rev. Chir., 63, 688 (Architecture interieure des vertebres)

MaXwell, J. C. (1853) 'On the equilibrium of elastic solids,' in 'Transactions of the Royal Society of Edinburgh,' Vol. XX, Part I, p. 87.

Vernon-Roberts, B., AND Pirie, C. J. (1973) Ann. rheum. Dis., 32, 406 (Healing trabecular microfractures in the bodies of lumbar vertebrae)

WolfF, J. (1870) Virchows Arch. path. Anat., 50, 389 (Ueber die Innere. Architektür der Knochen und ihre Beduetung für die Frage. von Knochen Wachstrum.) 